\title{
INDICADORES DE SUSTENTABILIDADE PARA MONITORAMENTO DE PROJETOS DE RECUPERAÇÃO DE ÁREAS DEGRADADAS
}

\author{
Thaiane Soares Montandon \\ Graduanda em Ciências Biológicas pela Universidade do Estado do Rio de Janeiro (UERJ) \\ \acmontandon@yahoo.com.br \\ Thereza Cristina Ferreira Camello \\ Doutora em Ciências Médicas - Biomédica - Microbiologista \\ Hospital Universitário Pedro Ernesto (UERJ/ OUERJ/ ONU/ UN-Habitat) - RJ - Brasil \\ Josimar Ribeiro de Almeida \\ Doutor - Professor Universidade do Estado do Rio de Janeiro (UERJ) \\ Pesquisador Senior Centro de Engenharia Nuclear/USP - RJ - Brasil
}

\section{Resumo:}

Nos últimos anos o crescimento da população e a ação do homem transformou diversos ecossistemas, aumentando significativamente a quantidade de áreas degradadas. Tendo em vista a necessidade de recuperar essas áreas, o Estado brasileiro determina a realização de Projetos de Recuperação de Àreas Degradadas (PRADs), que incluem monitoramento e avaliação da área. Este artigo teve como base duas formas de monitoramento, a proposta SER (2004) que apresenta diretrizes universais de recuperação, 9 atributos de áreas restauradas e 3 estratégias de avaliação, embora não apresente indicadores RAD; e a proposta de MELO et al., (2010) que apresenta uma Matriz de Avaliação de Projetos de Restauração, com 7 indicadores RAD. Os indicadores de sustentabilidade para essas áreas foram definidos por meio do Método Delphi. A aplicação da matriz de indicadores de sustentabilidade é um importante instrumento de planejamento, monitoramento e avaliação de áreas degradadas e a utilização do método Delphi para a construção da matriz garante um trabalho de monitoramento e avaliação mais eficiente e completo.

Palavras-chave: Recuperação de áreas degradadas; Monitoramento; Matriz de indicadores; Método Delphi.

\section{MATRIX OF SUSTAINABILITY INDICATORS FOR MONITORING AND EVALUATION OF DEGRADED AREAS RECOVERY}

\begin{abstract}
:
In recent years the population growth and the action of man turned many ecosystems, significantly increasing the amount of degraded areas. In view of the need to recover these areas, the Brazilian government determines the realization of areas Recovery of Degraded Projects (PRADs), including monitoring and evaluation of the area. This article was based on two forms of monitoring, the proposed SER (2004) that features universal recovery
\end{abstract}


guidelines, 9 attributes of restored areas and 3 assessment strategies, although it has not RAD indicators;. And the proposed MELO et al. (2010) which presents a Restoration Project Evaluation Matrix, with 7 RAD indicators. Sustainability indicators for these areas were defined through the Delphi Method. The application of sustainability indicators matrix is an important tool for planning, monitoring and evaluation of degraded areas and the use of the Delphi method to build the matrix ensures a monitoring work and more efficient and complete evaluation.

Keywords: recovery of degraded areas; Monitoring; Indicators matrix; Delphi Method.

\title{
INDICADORES DE SOSTENIBILIDAD PARA ÁREAS DE SEGUIMIENTO DEL PROYECTO DE RECUPERACIÓN
}

\begin{abstract}
Resumen
En los últimos años el crecimiento de la población y la acción del hombre se volvieron muchos ecosistemas, incrementando significativamente la cantidad de áreas degradadas. En vista de la necesidad de recuperar estas áreas, el gobierno brasileño determina la realización de zonas de recuperación de Proyectos Degradadas (Prads), incluyendo el monitoreo y evaluación de la zona. Este artículo se basa en dos formas de monitoreo, la SER propuesto (2004), que cuenta con directrices de recuperación universales, 9 atributos de las áreas restauradas y 3 estrategias de evaluación, aunque tiene indicadores no RAD ;. Y la propuesta MELO et al. (2010), que presenta un Proyecto Matriz de Evaluación de la Restauración, con 7 indicadores RAD. Los indicadores de sostenibilidad para estas áreas se definieron a través del Método Delphi. La aplicación de la matriz de indicadores de sostenibilidad es una herramienta importante para la planificación, seguimiento y evaluación de las áreas degradadas y el uso del método Delphi para construir la matriz asegura un trabajo de monitoreo y evaluación más eficiente y completa.
\end{abstract}

Palabras clave: la recuperación de áreas degradadas; Monitoreo; Matriz de indicadores; Método Delphi.

\section{INTRODUÇÃO}

A restauração ecológica vem se tornando referência na recuperação de áreas degradadas. É indispensável o monitoramento e avaliação dessa recuperação, com a finalidade de avaliar através de diversas ações se os objetivos propostos estão sendo alcançados. É necessário definir a trajetória ambiental da área em recuperação assim como a sua capacidade futura de sustentabilidade (HOWELL et al., 2012). São através de indicadores ecológicos que essa capacidade de sustentabilidade e a eficiência das medidas adotadas no processo de recuperação são avaliadas.

A recuperação consiste na restituição de um ecossistema podendo ser diferente da condição original, enquanto que a restauração é a restituição de um ecossistema o mais 
próximo possível da sua condição original. É importante ressaltar que a restauração é a melhor opção, quanto mais próxima da área original menores serão os impactos para a biodiversidade, para as relações interespecíficas e para as áreas próximas, porem nem sempre a restauração é viável (SER, 2004).

Há uma grande dificuldade ainda nesse processo de monitoramento e avaliação dos Projetos de Recuperação de Áreas Degradadas (PRADs) decorrente da visão das empresas como sendo apenas o formal comprimento da lei essas etapas (FUGIMOTO et al., 2004) Atualmente esse cenário vem mudando devido ao aumento das exigências normativas e fiscalização pelos órgãos ambientais, consequente da educação e valorização ambiental. Há ainda uma grande dificuldade em relação especifica ao monitoramento, o tempo e os recursos financeiros disponíveis normalmente são escassos para o monitoramento de grandes áreas de recuperação.

Os estudos e matrizes elaboradas para a área de monitoramento e avaliação são, em sua maioria, para áreas especificas. $\mathrm{O}$ estudo realizado tem como objetivo a montagem de uma matriz básica com indicadores de sustentabilidade para áreas em recuperação de forma geral, independente do clima e tipo de vegetação da área. As variáveis selecionadas para a produção dos indicadores têm como objetivo integrar as diferentes dimensões da sustentabilidade e foram baseadas em duas propostas, a proposta SER (2004) e a proposta de MELO et al., 2010.

\section{METODOLOGIA}

Inicialmente foi feito um estudo e análise das propostas utilizadas como base. A proposta SER (2004) define nove atributos de ecossistemas restaurados:

1) contém um conjunto característico de espécies que ocorrem no ecossistema de referencia;

2) consiste em espécies indígenas até o máximo grau possível;

3) todos os grupos funcionais necessários para o desenvolvimento e estabilidade do ecossistema restaurado encontram-se presentes; 
4) possui capacidade para suportar as populações reprodutivas das espécies necessárias para a sua estabilidade;

5) funciona de modo normal, sem disfunções;

6) foi integrado adequadamente com a matriz ecológica, com a qual interage através de fluxos e intercâmbios bióticos e abióticos;

7) as ameaças a saúde e integridade foram eliminadas ou reduzidas ao máximo;

8) suficientemente resiliente para suportar os eventos periódicos normais de estresse;

9) auto-sustentável, no mesmo grau do ecossistema de referência.

A proposta SER (2004) apresenta ainda três estratégias fundamentais que não podem ser ignoradas. São elas a análise dos atributos; a comparação direta da área em recuperação com a área de referência, observando todos os parâmetros descritos nos atributos e definir quais valores serão considerados como concluído o processo de recuperação e a análise de toda a trajetória do processo de recuperação.

A proposta de MELO et al., 2010 sugere uma matriz de avaliação de projetos de restauração com reflorestamento heterogêneo, apresentando sete indicadores de recuperação de áreas degradadas. Sendo os indicadores; cercamento; proteção de perturbações; mortalidade; ataque de formigas; mato competição na coroa das mudas; mato competição na entrelinha e cobertura de copas. Esses indicadores foram definidos com base em algumas diretrizes que consistem na adoção de indicadores relevantes e sensíveis a alterações, facilidade de coleta de informação de campo por qualquer técnico e indicação de manejo relacionada a diferentes níveis de adequação a cada reflorestamento. Essa proposta ainda define três níveis de adequação, sendo necessária a avaliação de em qual deles a área se encontra e apresentar ainda o valor encontrado. Com base nesse nível de adequação são definidas recomendações e a periodicidade do monitoramento.

Para a montagem da matriz, além das propostas base já descritas anteriormente, foi utilizado o método Delphi que consiste basicamente em um consenso das avaliações e opiniões de diversos especialistas, supondo que um julgamento baseado em diversas opiniões é mais completo e adequado. A matriz é composta por dimensão de sustentabilidade, 
perguntas-chave, indicador, descritor e nota. Podem apresentar ainda peso, dependendo da opinião dos especialistas e das características particulares de cada área de recuperação.

As matrizes preliminares foram feitas e enviadas para diversos especialistas, que no método Delphi pode variar de três até mais de cem especialistas envolvidos. É necessário que haja um consenso na opinião dos especialistas, cujos níveis podem variar de $50 \%$ a $80 \%$, nesse estudo utilizamos o nível de consenso de 50\%. As matrizes foram enviadas para os especialistas por email, ao serem respondidas foram analisadas. Aquelas que não apresentaram um consenso em primeiro momento, foram reenviadas para os especialistas com o objetivo de se atingir um nível de consenso satisfatório.

\section{RESULTADOS}

A matriz final está ilustrada nos quadros a seguir, considerando-se os dados referentes às dimensões política e tecnológica; econômica e financeira ambiental e ecológica, juntamente com o conhecimento a respeito da área e inclusão social da mesma.

Quadro 1 - Dimensão de dados políticos e tecnológicos

\begin{tabular}{|c|c|c|c|c|}
\hline DIMENSÃO & $\begin{array}{l}\text { PERGUNTA- } \\
\text { CHAVE }\end{array}$ & INDICADOR & DESCRITOR & NOTA \\
\hline \multirow{7}{*}{ Politica } & \multirow{7}{*}{$\begin{array}{c}\text { Está em } \\
\text { consonância com } \\
\text { a politica } \\
\text { ambiental? }\end{array}$} & \multirow{3}{*}{ PRADs (I1a) } & $\begin{array}{l}\text { Possui um PRADs } \\
\text { completo }\end{array}$ & 5 \\
\hline & & & $\begin{array}{l}\text { Possui um PRADs } \\
\text { parcial }\end{array}$ & 3 \\
\hline & & & $\begin{array}{c}\text { Não possui } \\
\text { PRADs }\end{array}$ & 1 \\
\hline & & \multirow{3}{*}{$\begin{array}{l}\text { Exigências impostas } \\
\text { (I1b) }\end{array}$} & $\begin{array}{c}\text { Atende mais de } \\
90 \% \text { das } \\
\text { exigências } \\
\end{array}$ & 5 \\
\hline & & & $\begin{array}{c}\text { Atende de } 50 \% \text { a } \\
90 \% \text { das } \\
\text { exigências }\end{array}$ & 3 \\
\hline & & & $\begin{array}{c}\text { Atende menos de } \\
50 \% \text { das } \\
\text { exigências } \\
\end{array}$ & 1 \\
\hline & & Fiscalização e & Periodicamente & 5 \\
\hline
\end{tabular}




\begin{tabular}{|c|c|c|c|c|} 
& & monitoramento (I1c) & $\begin{array}{c}\text { No inicio, meio e } \\
\text { termino }\end{array}$ & 3 \\
\cline { 4 - 5 } Subtotal máximo & & & Apenas no termino & 1 \\
\hline Tecnológica & $\begin{array}{c}\text { Observa os } \\
\text { princípios da } \\
\text { tecnologia } \\
\text { apropriada? }\end{array}$ & $\begin{array}{c}\text { Utilização de } \\
\text { equipamentos para } \\
\text { restauração (I2a) }\end{array}$ & Em algumas & $\mathbf{1 5}$ \\
\cline { 4 - 5 } & & & Em nenhuma & 1 \\
\hline Subtotal máximo & & & 5 \\
\hline
\end{tabular}

Quadro 2 - Dimensão Econômica/Financeira

\begin{tabular}{|c|c|c|c|c|}
\hline DIMENSÃO & $\begin{array}{l}\text { PERGUNTA- } \\
\text { CHAVE }\end{array}$ & INDICADOR & DESCRITOR & NOTA \\
\hline \multirow{9}{*}{$\begin{array}{l}\text { Econômica/ } \\
\text { financeira }\end{array}$} & \multirow{9}{*}{$\begin{array}{l}\text { Há condições } \\
\text { financeiras? }\end{array}$} & \multirow{3}{*}{$\begin{array}{l}\text { Recursos financeiros } \\
\text { para manutenção da } \\
\text { restauração (I3a) }\end{array}$} & Suficientes & 5 \\
\hline & & & Poucos & 3 \\
\hline & & & Nenhum & 1 \\
\hline & & \multirow{3}{*}{$\begin{array}{l}\text { Recursos financeiros } \\
\text { suficientes para a } \\
\text { restauração a condição } \\
\text { idêntica a original (I3b) }\end{array}$} & Total & 5 \\
\hline & & & Parcial & 3 \\
\hline & & & Nenhum & 1 \\
\hline & & \multirow{3}{*}{$\begin{array}{l}\text { Recursos financeiros } \\
\text { suficientes para o } \\
\text { tempo da restauração } \\
\text { (I3c) }\end{array}$} & Tempo mínimo & 5 \\
\hline & & & Tempo médio & 3 \\
\hline & & & Tempo máximo & 1 \\
\hline Subtotal máximo & & & & 15 \\
\hline \multirow{4}{*}{ Ambiental ecológica } & \multirow{4}{*}{$\begin{array}{c}\text { Recuperação total } \\
\text { da área? }\end{array}$} & \multirow{3}{*}{$\begin{array}{l}\text { Extensão da área } \\
\text { recuperada (I4a) }\end{array}$} & Mais de $90 \%$ & 5 \\
\hline & & & De $50 \%$ a $90 \%$ & 3 \\
\hline & & & Menos de $50 \%$ & 1 \\
\hline & & $\begin{array}{c}\text { Restauração das } \\
\text { espécies presentes na }\end{array}$ & Mais de $90 \%$ & 5 \\
\hline
\end{tabular}




\begin{tabular}{|c|c|c|c|}
\hline \multirow[t]{8}{*}{ 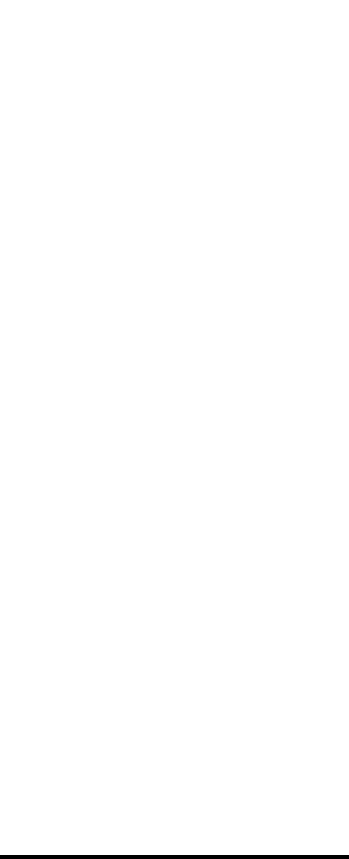 } & \multirow[t]{2}{*}{ condição original (I4b) } & De $50 \%$ a $90 \%$ & 3 \\
\hline & & Menos de $50 \%$ & 1 \\
\hline & \multirow{3}{*}{$\begin{array}{l}\text { Resiliência do novo } \\
\text { ecossistema (I4c) }\end{array}$} & Máxima & 5 \\
\hline & & Mediana & 3 \\
\hline & & Baixa & 1 \\
\hline & \multirow{3}{*}{$\begin{array}{l}\text { Media de Diversidade } \\
\text { de espécies (I4d) }\end{array}$} & $\begin{array}{c}\text { Mais de } 10 \\
\text { espécies por metro } \\
\text { quadrado }\end{array}$ & 5 \\
\hline & & $\begin{array}{c}\text { De } 5 \text { a } 10 \text { espécies } \\
\text { por metro } \\
\text { quadrado }\end{array}$ & 3 \\
\hline & & $\begin{array}{c}\text { Menos de } 5 \\
\text { espécies por metro } \\
\text { quadrado }\end{array}$ & 1 \\
\hline Subtotal máximo & & & 20 \\
\hline
\end{tabular}

Quadro 3 - Dimensão Conhecimento a respeito da área e inclusão social da mesma

\begin{tabular}{|c|c|c|c|c|}
\hline DIMENSÃO & $\begin{array}{l}\text { PERGUNTA- } \\
\text { CHAVE }\end{array}$ & INDICADOR & DESCRITOR & NOTA \\
\hline \multirow{6}{*}{ Conhecimento } & \multirow{6}{*}{$\begin{array}{l}\text { Há estudo e } \\
\text { conhecimento da } \\
\text { área original? }\end{array}$} & \multirow{3}{*}{$\begin{array}{c}\text { Conhecimento das } \\
\text { interações bióticas e } \\
\text { abióticas da área (I5a) }\end{array}$} & Total & 5 \\
\hline & & & Parcial & 3 \\
\hline & & & Mínimo & 1 \\
\hline & & \multirow{3}{*}{$\begin{array}{l}\text { Conhecimento das } \\
\text { melhores metodologias } \\
\text { de plantio e manejo } \\
\text { (I5b) }\end{array}$} & Total & 5 \\
\hline & & & Parcial & 3 \\
\hline & & & Mínimo & 1 \\
\hline Subtotal máximo & & & & 10 \\
\hline \multirow{2}{*}{ Inclusão social } & \multirow{2}{*}{$\begin{array}{l}\text { Há Inclusão da } \\
\text { população local } \\
\text { na recuperação? }\end{array}$} & \multirow{2}{*}{$\begin{array}{l}\text { Mao de obra da } \\
\text { população na } \\
\text { recuperação (I6a) }\end{array}$} & $\begin{array}{l}\text { Mais de } 70 \% \text { dos } \\
\text { cargos da } \\
\text { população local }\end{array}$ & 5 \\
\hline & & & $\begin{array}{c}\text { De } 30 \% \text { a } 70 \% \text { dos } \\
\text { cargos da } \\
\text { população local }\end{array}$ & 3 \\
\hline
\end{tabular}




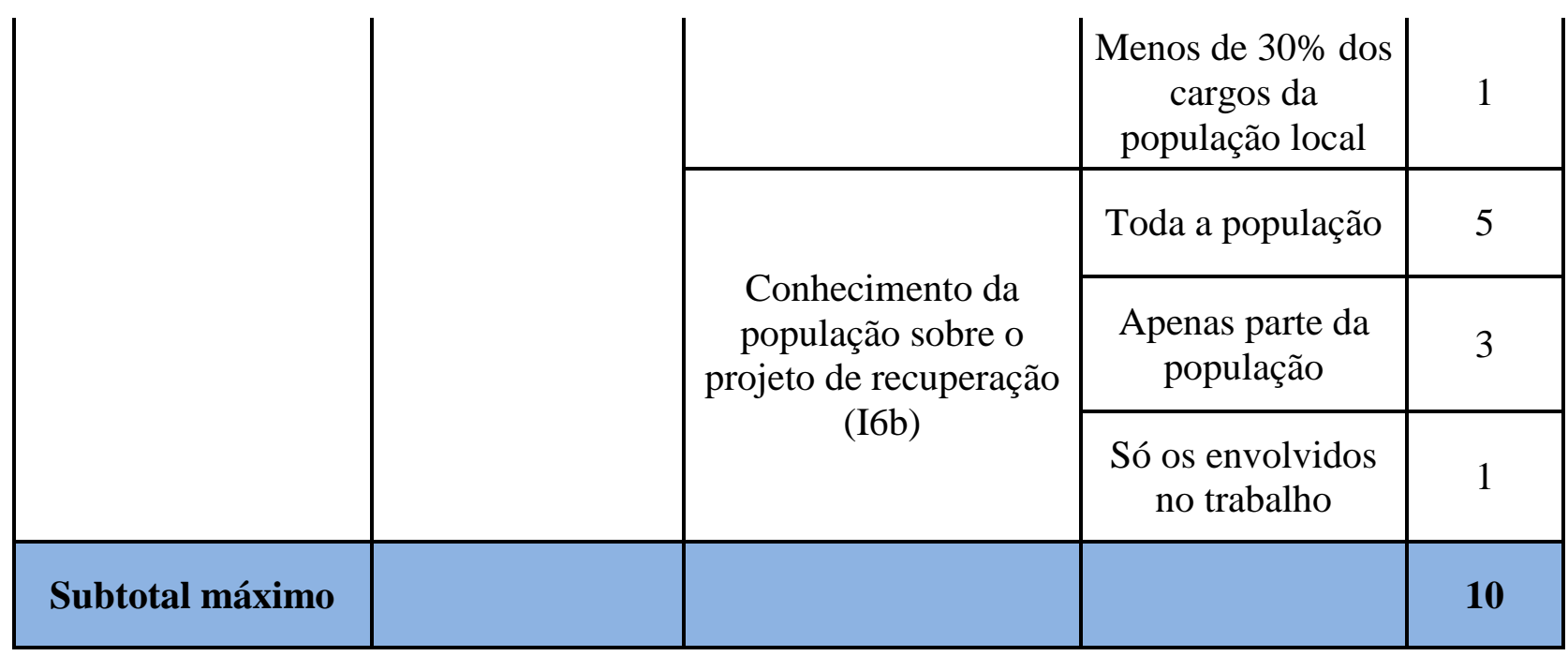

O nível de sustentabilidade da recuperação da área degradada é calculado pela seguinte fórmula:

$$
N S=\frac{\sum \text { das notas obtidas na avaliacao }}{\sum \text { da maxima pontuacao em cada dimensao }} \times 10^{-1}
$$

A Tabela 1 representa os níveis de sustentabilidade determinados pelos intervalos de sustentabilidade que variam segundo a aplicação da fórmula acima, entre valores de 0 a 10 .

Tabela 1 - Intervalo e nível de sustentabilidade

\begin{tabular}{cc}
\hline Intervalo de sustentabilidade & Nível de sustentabilidade \\
\hline 0 & INSUSTENTAVEL \\
$1 \leq \mathrm{NS} \leq 4$ & BAIXA SUSTENTABILIDADE \\
$5 \leq \mathrm{NS} \leq 8$ & MEDIA SUSTENTABILIDADE \\
$9 \geq \mathrm{NS} \leq 10$ & ALTA SUSTENTABILIDADE \\
\hline
\end{tabular}

\section{DISCUSSÃO E CONSIDERAÇÕES FINAIS}

O estudo apresenta uma tabela básica aplicável a qualquer área de recuperação, reforçando apenas que os pesos de cada indicador devem ser estipulados com base nas características específicas de cada área analisada. Essas características influenciam 
diretamente no processo de recuperação. Por exemplo áreas em processo de recuperação próximas a áreas habitacionais necessitam de maiores medidas relacionadas a população local, de modo que os indicadores referentes a essa característica devem receber um peso maior. Da mesma forma, áreas que antes apresentavam grande biodiversidade não podem ser recuperadas de forma homogênea, há uma necessidade ainda maior de estudos para uma restauração heterogênea, com diferentes espécies de forma a manter a biodiversidade local original e preservar ao máximo as relações existentes anteriormente. Nesse caso os indicadores relacionados à heterogeneidade da área apresentam um peso maior. Como descrito anteriormente as dificuldades em relação ao tempo e aos recursos financeiros na amostragem de grandes áreas em recuperação impedem um melhor estudo das características da área e consequentemente dificultam a escolha de indicadores de sustentabilidade e seus pesos. Todos os indicadores e medidas devem ser escolhidos visando o menor impacto ao ambiente como um todo.

O cálculo do nível de sustentabilidade é fundamental para a avaliação do processo de recuperação da área, visto que áreas recuperadas devem ser autossustentáveis. O projeto de recuperação de área degradada (PRAD) pode ser considerado concluído e completo quando a área se encontrar no nível de alta sustentabilidade.

A aplicação da matriz de indicadores de sustentabilidade é um importante instrumento de planejamento, monitoramento e avaliação de áreas degradadas e a utilização do método Delphi para a construção da matriz garante um trabalho de monitoramento e avaliação mais eficiente e completo, tendo em vista que engloba a opinião e consenso de diversos especialistas da área nas diversas dimensões.

\section{REFERÊNCIAS BIBLIOGRÁFICAS}

ANDRADE, Gilberto Fugimoto de; SANCHEZ, Gabriela Fernandez; ALMEIDA, Josimar Ribeiro de. Monitoramento e Avaliação em Projetos de Recuperação de Áreas Degradadas. Revista Internacional de Ciências, Rio de Janeiro, v. 4, n. 2, p.13-26, nov. 2014. DOI: 10.12957/ric.2014.13833. Disponível em: <http://www.epublicacoes.uerj.br/index.php/ric/article/view/13833>. Acesso em: 15 jun. 2015.

HOWELL, Evelyn A.; HARRINGTON, John A.; GLASS, Stephen B.. Introduction to Restoration Ecology. Washington: Island Press, 2012. 436 p. 
REIS, Claudia Macedo; RESENDE, Roberto Ulisses; MELO, Antônio Carlos Galvão de. Guia para monitoramento de reflorestamentos para restauração. Circular Técnica 1 Projetos Mata Ciliar. São Paulo: Secretaria de Meio Ambiente, 2010. Disponível em: $<$ http://www.terrabrasilis.org.br/ecotecadigital/index.php?option=com_abook\&view=book\&c atid=8:monitoramento\&id=286: guia-para-monitoramento-de-reflorestamentos-pararestauracao>. Acesso em: 15 jun. 2015.

SANTIAGO, Leila Santos; DIAS, Sandra Maria Furiam. Matriz de indicadores de sustentabilidade para a gestão de resíduos sólidos urbanos. Engenharia Sanitária e Ambiental, Rio de Janeiro, v. 17, n. 2, p.203-212, 2012. DOI: 10.1590/s141341522012000200010. Disponível em: <http://www.scielo.br/scielo.php?script=sci_arttext\&pid=S1413-41522012000200010>. Acesso em: 15 jun. 2015.

SER - SOCIETY FOR ECOLOGICAL RESTORATION INTERNATIONAL. Grupo de Trabalho Sobre Ciência e Política. Princípios da SER Internacional sobre Restauração Ecológica. www.ser.org y Tucson, 2004. Tradução de Griffith J. J.. Disponível em: <http://www.ser.org/docs/default-document-library/ser-primer-portuguese.pdf $>$. Acesso em: 15 jun. 2015. 